\title{
CARACTERIZAÇÃO FÍSICA, QUÍMICA E TÉRMICA DE ENDOCARPOS DE BUTIÁS
}

\author{
V. ROSSETO ${ }^{1}$, R. ZOTTIS ${ }^{1}$, M. M. MORAIS ${ }^{1}$ e A. R. F. de ALMEIDA ${ }^{1}$ \\ ${ }^{1}$ Universidade Federal do Pampa, Campus Bagé \\ E-mail para contato: marcilio.morais@unipampa.edu.br
}

\begin{abstract}
RESUMO - Atualmente tem sido avaliada a utilização de uma série de resíduos agroindustriais para a produção de carvão ativado, devido à sua grande disponibilidade, baixo custo e por serem materiais renováveis. Assim, o objetivo do presente trabalho foi realizar a caracterização morfológica, física, química e térmica de endocarpos moídos de frutos de butiás (Butia quaraimana), com vistas à produção de

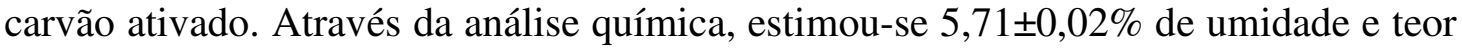
de cinzas de $0,65 \pm 0,03 \%$. Verificou-se na análise física que a massa específica real e aparente foi de $1,461 \pm 0,001 \mathrm{~g} / \mathrm{cm}^{3}$ e $0,738 \pm 0,017 \mathrm{~g} / \mathrm{cm}^{3}$, respectivamente. A porosidade do leito de partículas foi de 0,495 e o diâmetro médio de Sauter foi de $0,59 \pm 0,04 \mathrm{~mm}$. A distribuição granulométrica indicou que aproximadamente $90 \%$ das partículas apresentaram de 0,5 a $1,5 \mathrm{~mm}$. Na análise termogravimétrica, avaliou-se a pirólise e a combustão de amostras de 0,945 e 0,113 $\mathrm{mm}$. As curvas de perda de massa apresentaram tendências semelhantes em ambos os processos e para as duas granulometrias de partículas. Observou-se que a perda de massa foi mais pronunciada nas partículas de menor diâmetro, em especial na pirólise, devido a menor resistência a transferência de massa em tais partículas. Destaca-se as partículas de 0,945 mm que foram mais estáveis termicamente na pirólise. A caracterização física, química, térmica indicou que o endocarpo de butiá é um bom precursor do carvão ativado.
\end{abstract}

\section{INTRODUÇÃO}

Em função da crescente preocupação ambiental, atualmente tem se observado um aumento do número de estudos que avaliam a utilização de produtos que possibilitam a remediação de danos ambientais. Dentre os materiais, destaca-se o carvão ativado, produto largamente utilizado no tratamento de água e efluentes e na purificação do ar e gases (Albuquerque Jr. et al., 2008; Bhatnagar e Sillanpää, 2010; Claudino, 2003). O carvão ativo pode ser produzido a partir de qualquer material carbonáceo, natural ou sintético (Cambuim, 2009; Rocha et al., 2006), sendo considerado um adsorvente universal por possuir alto grau de porosidade e elevada área superficial interna (Bhatnagar e Sillanpää, 2010).

Para a produção de carvão ativado tem sido avaliada uma série de resíduos agroindustriais, em função da sua grande disponibilidade, baixo custo e por serem materiais renováveis (Bhatnagar e Sillanpää, 2010). Dentre os resíduos lenhosos estão os caroços (endocarpos lenhosos) de diversos frutos, como amêndoa; avelã; azeitona, coco da baía; damasco; macadâmia; noz; pêssego, entre outros (Albuquerque Jr. et al., 2008; Aygün et al., 2003; Cambuim, 2009; Diniz et al., 2004; El-Sheikh et al., 2004; Maroto-Valer et al., 2004; Rocha et al,. 2006). Materiais ligninocelulósicos são muito porosos e tem elevada área superficial que permite o contato de soluções aquosas com 
os componentes das paredes celulares; além disso, a rigidez da lignina evita que as partículas de carvão ativado rompam-se (Cambuim, 2009; Pehlivan et al., 2009).

Butiás são frutos de uma palmeira conhecida como butiazeiro, com ocorrência natural no Brasil, Argentina, Paraguai e Uruguai (Deble et al., 2011). No Brasil, atualmente há 17 espécies catalogadas, distribuídas principalmente nos Biomas Cerrado, Mata Atlântica e Pampa, sendo que a produção de butiás ocorre de forma extrativista (Deble et al., 2011; Faria et al., 2008a). A polpa (epicarpo e mesocarpo) do butiá é consumida in natura e utilizada na produção de bebidas e doces, sendo rica em vitaminas e sais minerais (Faria et al., 2008a; Sganzerla, 2010). Apresentam de 1-3 sementes, que também são comestíveis, com alto teor de fibras, fenóis e lipídios, em especial ácidos graxos saturados (Faria et al., 2008b; Sganzerla, 2010). O endocarpo, caroço que envolve as sementes, é lenhoso, sendo descartado durante a produção artesanal e agroindustrial de produtos à base de butiá.

A espécie Butia quaraimana Deble \& Marchiori é endêmica do Palmar do Coatepe, região rural localizada em Quaraí/RS (Deble et al., 2012). Em uma área de aproximadamente $42 \mathrm{~km}^{2}$, há a ocorrência natural de milhares de butiazeiros e são desenvolvidas atividades extrativistas dos frutos e folhas de tal espécie pelos moradores da região (Deble et al.; 2012; Rosseto et al., no prelo). A produtividade média dos butiazeiros da espécie $B$. quaraimana é de $5,08 \mathrm{~kg}$ de butiás por butiazeiro e 13,70\% do peso do fruto corresponde ao peso do endocarpo (Rosseto et al., no prelo). Com a finalidade de se avaliar o potencial para a produção de carvão ativado utilizando um resíduo agrícola, o objetivo do trabalho foi realizar a caracterização morfológica, física, química e térmica de endocarpos de B. quaraimana.

\section{MATERIAL E MÉTODOS}

\subsection{Matéria-prima}

No presente trabalho foram utilizados butiás de $B$. quaraimana. Coletou-se um cacho de cada butiazeiro (30 no total). Os frutos foram debulhados, lavados e despolpados manualmente. Após, os pirênios (endocarpo e sementes) foram lavados, secos em temperatura ambiente, armazenados em sacos plásticos e refrigerados em freezer $\left(-18^{\circ} \mathrm{C}\right)$ até a utilização. Para a separação dos endocarpos e sementes foram utilizadas morsas.

Para todas as análises, com exceção da caracterização morfológica, foram utilizados endocarpos moídos. Como o material é lenhoso, portanto, de difícil moagem, os endocarpos foram previamente quebrados em prensa hidráulica (pressão de cerca de 20 toneladas por aproximadamente 10 segundos) até que o diâmetro fosse menor do que 8,0 $\mathrm{mm}$, tamanho máximo de partícula que poderia ser colocado em um moinho de facas. Para a redução de tamanho das partículas previamente quebradas, o endocarpo foi moído em um moinho de facas durante 15 segundos sem peneira e moído novamente por 1 minuto com peneira de 30 mesh.

\subsection{Procedimento experimental}

Foi realizada a caracterização morfológica dos endocarpos de butiás não moídos e moídos, utilizando-se estereomicroscópio (Motic, K500).

Para a caracterização química dos endocarpos moídos, foi avaliado o teor de umidade e de 
cinzas em triplicada pelo método da AOAC (1997). O teor de umidade foi analisado em estufa de secagem a $105^{\circ} \mathrm{C}$ durante $24^{\circ} \mathrm{C}$. Já o teor de cinzas foi avaliado em forno mufla a $550^{\circ} \mathrm{C}$.

A caracterização física abrangeu a determinação dos seguintes parâmetros: massa específica real, massa específica aparente, porosidade do leito de partículas, distribuição granulométrica e diâmetro médio de Sauter. A massa específica real $\left(\rho_{\text {real }}\right)$ foi avaliada através de picnometria gasosa, utilizando o picnômetro a gás hélio (Quantachrome Instruments, UPY-30F). A massa específica aparente $\left(\rho_{a p}\right)$ foi determinada através de ensaio de proveta. A porosidade do leito de partículas $(\varepsilon)$ foi obtida por meio da relação entre $\rho_{\text {real }}$ e $\rho_{a p}$.

Para a obtenção da distribuição granulométrica das partículas, foram realizados ensaios de peneiramento em duplicata, utilizando-se um jogo de peneiras de 3,360 a 0,038 mm, (com um fundo cego para coletar os finos), acoplado em um agitador (Bertel), durante 15 minutos a um nível de agitação 10. A partir desses dados foi determinado o diâmetro médio de Sauter $\left(D_{S}\right)$.

Após a classificação granulométrica foram selecionadas partículas de tamanhos diferentes $(0,945$ e $0,113 \mathrm{~mm})$, com o objetivo de se avaliar possíveis diferenças dos materiais em relação às características morfológicas, físicas e térmicas.

A caracterização térmica foi realizada por análise termogravimétrica (TGA) tanto em atmosfera inerte com nitrogênio gasoso $(10 \mathrm{~mL} / \mathrm{min})$, quanto em atmosfera oxidante em contato direto com o ar ambiente (fluxo natural). Utilizou-se o equipamento TGA 50, marca Shimadzu, a $10^{\circ} \mathrm{C} / \mathrm{min}$, com duas rampas de aquecimento (até $110^{\circ} \mathrm{C}$ por $15 \mathrm{~min}$ e até $900^{\circ} \mathrm{C}$ por $15 \mathrm{~min}$ ). Foi utilizada aproximadamente $13 \mathrm{mg}$ de material em cada análise.

\section{RESULTADOS E DISCUSSÃO}

A morfologia de endocarpos de butiás não moídos é apresentada na Figura 1. Observou-se que tal biomassa apresenta uma estrutura heterogênea, sendo que a porção mais rígida do material (coloração marrom-escuro) é entremeada por fibras (coloração mais clara). Além disso, há uma camada na porção mais interna do endocarpo também com coloração mais clara de aspecto fibroso. Os endocarpos de $B$. quaraimana apresentam em média diâmetro longitudinal de $21,03 \pm 2,45 \mathrm{~mm}$ e diâmetro equatorial de $11,69 \pm 1,43 \mathrm{~mm}$ (Rosseto et al., no prelo).
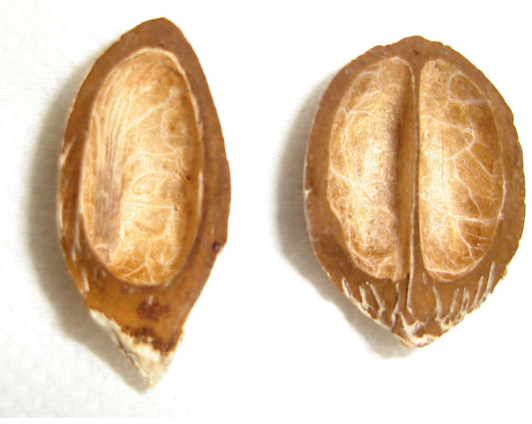

Figura 1 - Imagens de endocarpos de butiás com lóculos para uma e duas sementes. 6,4 x.

Os resultados da caracterização química, assim como a comparação com dados da literatura, são apresentados na Tabela 1. Verificou-se que os endocarpos de butiás apresentaram baixo teor 
de umidade e cinzas, semelhante ao apresentado por endocarpos lenhosos de outras espécies. Moreno-Castilla, 2004 afirma que as cinzas podem prejudicar o processo de adsorção, pois podem bloquear a porosidade da matriz carbônica, adsorvendo preferencialmente água, em função de sua natureza hidrofílica. Além disso, em geral, o carvão ativado é composto de 72 a $90 \%$ de carbono (Haimour e Emeish, 2006). O baixo teor de cinzas, assim como a elevada concentração de carbono encontrado no endocarpo de butiá, mesmo sem ativação, indicam que o material possui potencial para produção de carvão ativado.

Tabela 1 - Caracterização química de endocarpos de butiás e compilação de dados da literatura para endocarpos de frutos de outras espécies

\begin{tabular}{|c|c|c|}
\hline Material & Umidade (\% & Cinzas (\%) \\
\hline Butiá (Autores, 2014) & $5,71 \pm 0,02$ & $0,65 \pm 0,03$ \\
\hline Amêndoa (Aygün et al., 2003; Pehlivan et al., 2009) & 8,7 & $0,31-0,76$ \\
\hline Avelã (Aygün et al., 2003; Pehlivan et al., 2009) & 7,7 & $0,49-1,46$ \\
\hline Azeitona (El-Sheikh et al., 2004) & - & 0,68 \\
\hline Damasco (Aygün et al., 2003) & 8,1 & 0,78 \\
\hline Macadâmia (Rocha et al., 2006) & 10,20 & 0,50 \\
\hline Noz (Aygün et al., 2003) & 11 & 0,44 \\
\hline
\end{tabular}

Na Tabela 2 são apresentados os resultados da massa específica e comparação com dados da literatura. A porosidade do leito das partículas foi de 0,495. Observou-se que a massa específica aparente foi semelhante à de endocarpos de frutos de outras espécies. Partículas irregulares e com baixa esfericidade tendem a formar um leito de partículas menos empacotado; este fato pode explicar o valor encontrado para a massa específica aparente e a porosidade de leito. Outros estudos devem ser realizados para se determinar a esfericidade das partículas, o que auxiliará no entendimento dos valores encontrados.

Tabela 2 - Massa específica real e aparente de endocarpos de butiás e compilação de dados da literatura para endocarpos de frutos de outras espécies

\begin{tabular}{|c|c|c|}
\hline Material & $\rho_{\text {real }}\left(\mathbf{g} / \mathbf{c m}^{\mathbf{3}}\right)$ & $\rho_{a p}\left(\mathbf{g} / \mathbf{c m}^{\mathbf{3}}\right)$ \\
\hline Butiá (Autores, 2014) & $1,461 \pm 0,001$ & $0,738 \pm 0,017$ \\
\hline Amêndoa (Aygün et al., 2003) & - & 0,800 \\
\hline Avelã (Aygün et al., 2003) & - & 0,790 \\
\hline Damasco (Aygün et al., 2003) & - & 0,820 \\
\hline Macadâmia (Rocha et al., 2006) & 1,015 & - \\
\hline Noz (Aygün et al., 2003) & - & 0,810 \\
\hline
\end{tabular}

Na Figura 2 é apresentada a distribuição granulométrica da amostra em questão; " $x_{i}$ " é a fração de partículas retida em uma determinada peneira e " $\mathrm{D}_{\mathrm{p}}$ " é o diâmetro médio de partícula referente ao diâmetro de malha da peneira passante e da peneira retida. Observou-se que 90\% das partículas encontram-se na faixa 0,5 a $1,5 \mathrm{~mm}$. O diâmetro médio de Sauter calculado foi de $0,59 \pm 0,04 \mathrm{~mm}$. 


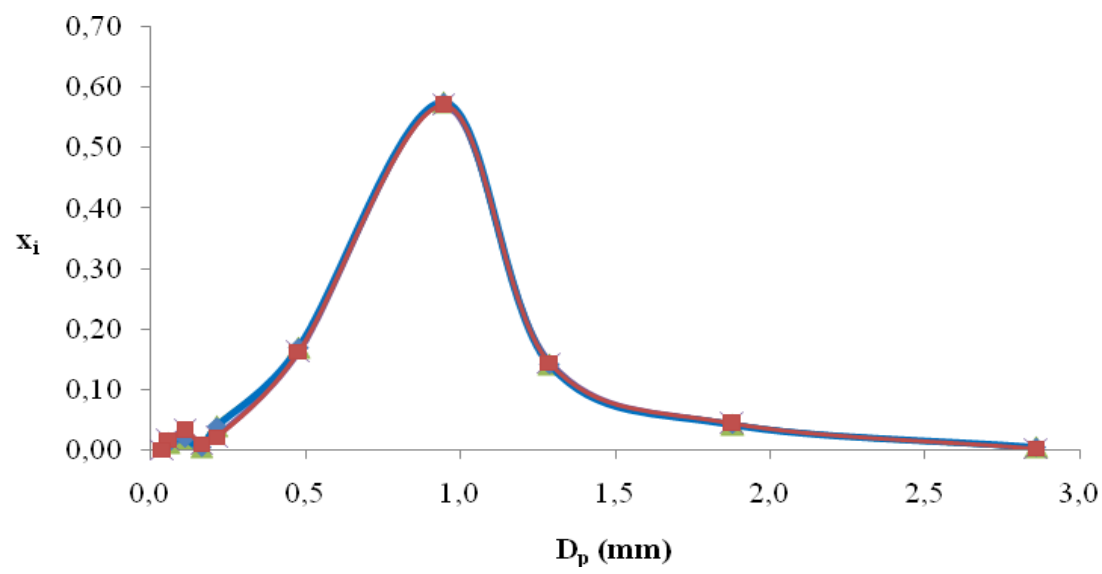

Figura 2 - Distribuição granulométrica diferencial de endocarpos moídos de butiá (duplicata do ensaio do peneiramento).

A morfologia de endocarpos de butiás moídos é apresentada na Figura 3. Verificou-se que as partículas apresentam morfologia irregular, o que está de acordo com os resultados encontrados na Tabela 2. Observou-se visualmente que as partículas maiores (Fig. 3a) são representadas principalmente pela porção mais rígida do endocarpo, enquanto que as partículas menores (Fig. 3b) são representadas pelo material fibroso mais claro.

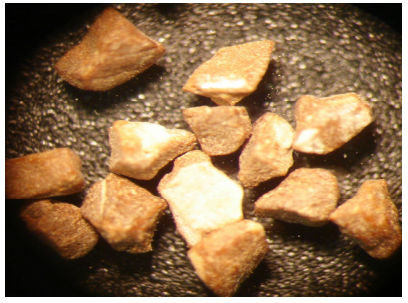

(a)

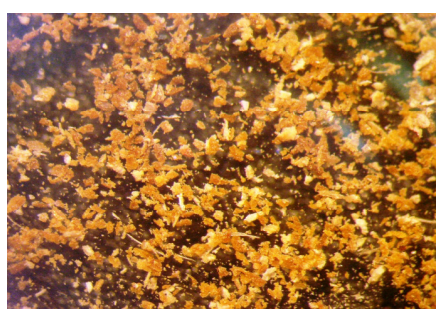

(b)

Figura 3 - Imagens de endocarpos moídos de butiás de 0,945 mm (a) e de 0,113 mm (b). 25 x.

Para a caracterização física das partículas de tamanhos diferentes foi avaliada a massa específica real. As partículas de $0,945 \mathrm{~mm}$ apresentaram $1,600 \pm 0,007 \mathrm{~g} / \mathrm{cm}^{3}$, enquanto que as partículas de $0,113 \mathrm{~mm}$ exibiram $1,474 \pm 0,002 \mathrm{~g} / \mathrm{cm}^{3}$. A explicação para esta diferença pode estar vinculada ao processo de moagem. Possivelmente durante o processo de moagem foram separadas porções de material com composição estrutural de tecidos vegetais diferentes, conforme também elucidado pela Figura 3.

Os resultados da análise termogravimétrica do endocarpo de butiá moído são apresentados na Figura 4. Comparando-se a combustão e a pirólise das amostras, as curvas termogravimétricas do mesmo processo apresentaram tendências semelhantes para as duas granulometrias das partículas. 


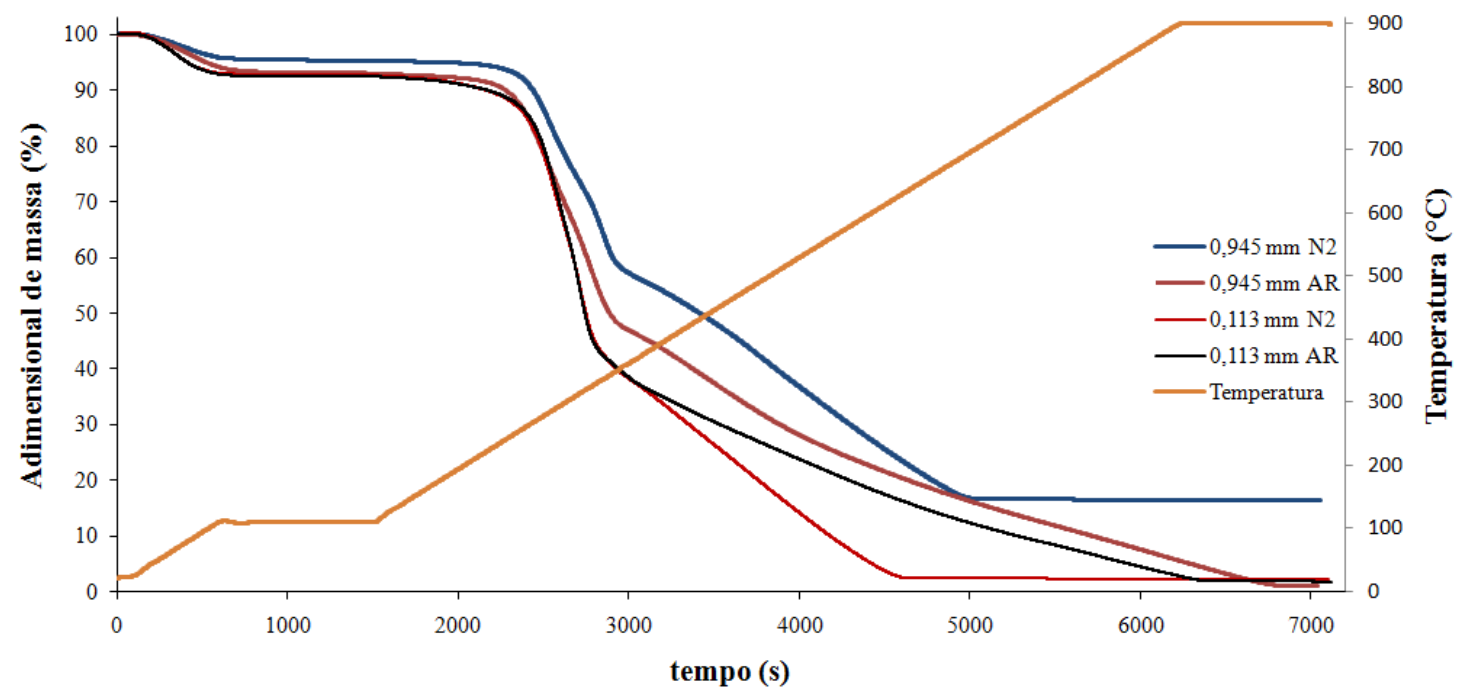

Figura 4 - Curvas termogravimétricas de pirólise e combustão de partículas de endocarpo de butiá de 0,945 e $0,113 \mathrm{~mm}$

Analisando-se as maiores perdas de massa durante a pirólise, observou-se perda de massa para as duas amostras ao redor de $6,0 \%$ até $110^{\circ} \mathrm{C}$; de $8,0-48,0 \%$ entre $235-350^{\circ} \mathrm{C}$; e de 48,0 $89,0 \%$ de $350-680^{\circ} \mathrm{C}$. Observou-se que as maiores partículas apresentaram-se mais estáveis termicamente, estabilizando-se em aproximadamente $700^{\circ} \mathrm{C}$ e apresentando maior porcentagem de resíduo (16\%) do que as partículas menores (2\%). Partículas de maior tamanho tendem a apresentar maior resistência à volatilização durante a pirólise do que partículas menores, o que está de acordo com os resultados encontrados para a massa final de cada um dos tamanhos de partícula.

Considerando-se a combustão, observou-se comportamento semelhante ao da pirólise até aproximadamente $300^{\circ} \mathrm{C}$. Verificou-se que a perda de massa para as duas amostras foi próxima a $7,3 \%$ até $110^{\circ} \mathrm{C}$; de $9,8-54,0 \%$ entre $237-346^{\circ} \mathrm{C}$; e de $54,0-1,3 \%$ entre $346-900^{\circ} \mathrm{C}$. Ambas as partículas somente foram estabilizadas a partir de $900^{\circ} \mathrm{C}$, sendo que a porcentagem de resíduo das partículas maiores foi de $0,9 \%$ e das partículas menores de $1,8 \%$.

Comparando-se o tamanho de partícula em ambos os processos, as amostras de 0,113 $\mathrm{mm}$ apresentaram o primeiro pico de perda de massa a $67^{\circ} \mathrm{C}$, enquanto que para as amostras de 0,945 $\mathrm{mm}$ este ocorreu a $77^{\circ} \mathrm{C}$. Observou-se que perda de umidade foi de 5 a $7 \%$ até $110^{\circ} \mathrm{C}$; tal

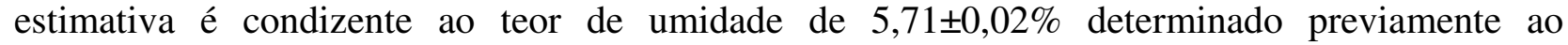
peneiramento das amostras. A derivada da curva termogravimétrica revelou a existência de dois picos na etapa de maior perda de massa das amostras: o primeiro a $293^{\circ} \mathrm{C}$, que pode estar associada à despolimerização da hemicelulose; e o segundo a $315^{\circ} \mathrm{C}$ (para as partículas menores) e a $340^{\circ} \mathrm{C}$ (para as partículas maiores), possivelmente relacionada com a decomposição da celulose e lignina (Martins et al., 2009). 


\section{CONCLUSÃO}

O endocarpo de butiá apresentou morfologia heterogênea com a porção mais rígida entremeada por fibras, em especial em sua camada interna. O material possui baixo teor de umidade e de cinzas, além de massa específica aparente semelhante a endocarpos de frutos de outras espécies. A distribuição granulométrica indicou que aproximadamente $90 \%$ das partículas apresentaram de 0,5 a $1,5 \mathrm{~mm}$. Comparando-se partículas de diferentes tamanhos $(0,945$ e 0,113 $\mathrm{mm})$, verificou-se que ambas exibiram formato irregular, sendo que as partículas maiores são formadas principalmente pela porção mais rígida, de cor marrom escura, enquanto que as partículas menores são representadas pelo material fibroso mais claro. As partículas de maior granulometria exibiram massa específica real superior a das partículas menores. As análises termogravimétricas indicaram comportamento térmico semelhante na pirólise e combustão para ambas as partículas. Destacam-se as partículas de $0,945 \mathrm{~mm}$ que foram mais estáveis termicamente na pirólise. As variações observadas para as partículas em questão podem estar associadas não apenas com o tamanho das partículas, mas com diferenças em relação à composição estrutural dos tecidos vegetais presentes no endocarpo. A caracterização física, química, térmica e indicaram que o endocarpo de butiá é um bom precursor do carvão ativado.

\section{REFERÊNCIAS}

AlbuQuerque JR., E. C.; MÉNDEZ, M. O. A.; COUTINHO, A. R.; FRANCO, T. T. Removal of cyanobacteria toxins from drinking water by adsorption on activated carbon fibers. Mater. Res., v. 11, p. 371-380, 2008.

AOAC - ASSOCIATION OF OFFICIAL ANALYTICAL CHEMISTS. Official methods of analysis of AOAC International. 16 ed. Gaitheersburg: AOAC, 1997.

AYGÜN, A.; YENISOY-KARAKAŞ, S.; DUMAN, I. Production of granular activated carbon from fruit stones and nutshells and evaluation of their physical, chemical and adsorption properties. Micropor. Mesopor. Mat., v. 66, p. 189-195, 2003.

BHATNAGAR, A.; SILLANPÄ̈̈, M.. Utilization of agro-industrial and municipal waste materials as potential adsorbents for water treatment - A review. Chem. Eng. J., v. 157, p. 277-296, 2010.

CAMBUIM, K. B. Carvão de endocarpo de coco da baía ativado quimicamente com H3PO4 e fisicamente com vapor d'água: produção, caracterização e aplicações. Tese (Doutorado em Química Analítica). Universidade Federal da Paraíba, João Pessoa, 2009.

CLAUDINO, A. Preparação de carvão ativado a partir de turfa e sua utilização na remoção de poluentes. Dissertação (Mestrado em Engenharia Química), Universidade Federal de Santa Catarina, Florianópolis, 2003.

DEBlE, L. P.; MARCHIORI, J. N. C.; ALVES, F. S.; OLIVEIRA-DEBLE, A. S. Survey on Butia (Arecaceae): from Rio Grande do Sul State (Brazil). Balduinia, v. 30, p. 3-24, 2011.

DEBlE, L. P.; MARCHIORI, J. N. C.; ALVES, F. S.; OLIVEIRA-DEBLE, A. S. Butia quaraimana (Arecaceae), uma nova espécie para o Rio Grande do Sul (Brasil). Balduinia, v. 33, p. 9-20, 2012.

DINIZ, J.; CARDOSO, A. L.; STAHL, J. A.; VILleTtI, M. A.; MARTINS, A. F. Poder calorífico da casca de arroz, caroço de pêssego, serragem de eucalipto e de seus produtos de 
pirólise. Ciênc. Nat., v. 26, p. 25-32, 2004.

EL-SHEIKH, A. H.; NEWMAN, A. P.; AL-DAFFAEE, H. K.; PHULL, S.; CRESSWELL, N. Characterization of activated carbon prepared from a single cultivar of Jordanian Olive stones by chemical and physicochemical techniques. J. Anal. Appl. Pyrolysis, v. 71, p. 151$164,2004$.

FARIA, J. P.; ALMEIDA, F.; SILVA, L. C. R.; VIEIRA, R. F.; AGOSTINI-COSTA, T. S. Caracterização da polpa do coquinho-azedo (Butia capitata var. capitata). Rev. Bras. Frutic., v. 30, p. 827-829, 2008a.

FARIA, J. P.; ARELLANO, D. B.; GRIMALDI, R.; SILVA, L. C. R.; VIEIRA, R. F.; SILVA, D. B.; AGOSTINI-COSTA, T. S. Caracterização química da amêndoa de coquinho-azedo (Butia capitata var. capitata). Rev. Bras. Frutic.,v. 30, p. 549-552, 2008 b.

HAIMOUR, N. M.; EMEISH, S. Utilization of date stones for production of activated carbon using phosphoric acid. Waste Manage., v. 26, p 651-660, 2006.

MAROTO-VALER, M. M.; DRANCA, I.; LUPASCU, T. NASTAS, R. Effect of adsorbate polarity on thermodesorption profiles from oxidized and metal-impregnated activated carbons. Carbon, v. 42, p. 2655-2659, 2004.

MARTINS, M. A.; MATTOSO, L. H. C.; PESSOA, J. D. C. Comportamento térmico e caracterização morfológica das fibras de mesocarpo e caroço do açaí. Rev. Bras. Frutic., v. 31, p. 1150-1157, 2009.

MORENO-CASTILLA, C. Adsorption of organic molecules from aqueous solutions on carbon materials. Carbon, v. 42, p. 83-94, 2004.

PEHLIVAN, E.; ALTUN, T.; CETIN, S.; BHANGER, I. Lead sorption by waste biomass of hazelnut and almond shell. J. Hard. Mater., v. 167, p. 1203-1208, 2009.

ROCHA, W. D.; LUZ, J. A. M.; LENA, J. C.; BRUÑA-ROMERO, O. Adsorção de cobre por carvões ativados de endocarpo de noz macadâmia e de semente de goiaba. R. Esc. Minas, v. 59, p. 409-414, 2006.

ROSSETO, V.; ROSA, C. S.; SILVA, K. O.; ZEPPENFELD, V. B. Biometria de frutos de Butia quaraimana no sudoeste do Rio Grande do Sul (no prelo).

SGANZERLA, M. 2010. Caracterização físico-química e capacidade antioxidante do butiá. Dissertação (Mestrado em Ciências). Universidade Federal de Pelotas, Pelotas, 2010. 